International Journal of Engineering \& Technology, $7(1.1)(2018) 704-705$
International Journal of Engineering \& Technology
SPC
Website www.sciencepubco.com/index.php/IJET
Research paper

\title{
Is Agile Cure for Everything
}

\author{
Rahul Agarwal $^{1 *}$, Dr. Anurupa Singh², Dr. Ambrish Joshi ${ }^{3}$ \\ ${ }^{1}$ Research Scholar, Amity University \\ ${ }^{2}$ Associate Professor, Amity University \\ ${ }^{3} A V P$, Genpact \\ *Email: rahullagarwal@gmail.com
}

\begin{abstract}
This research paper explores Agile- more in terms of its successes, its limitations, myths around Agile and where it could be the best fit. The paper also envisions to demystify the aura around Agile methodology with excerpts gathered from discussions with some of the Agile thought leaders.
\end{abstract}

Keywords: Agile Methodology, Agile Projects, Limitations and Challenges, Agile cure

\section{Introduction}

Project not shaping up- Agile, Requirements not fixed- Agile, Early Delivery- Agile, Project delayed- Agile, Productivity problem- Agile, Technical issues- Agile, Customer not responsiveAgile, Customer aggressive- Agile, Industry standard- Agile, Growth sluggish- Agile. Agile, Agile, Agile...it has become such a misused word that if not corrected it would lose its essence. After all what is Agile. Is it really worth going the Agile way? Can it cure all the Industry problems? How can it be leveraged? Shall we blindly implement Agile. Shall we use inhouse people or hire an external consultant. How much time does it take for a company to implement Agile...questions are endless, and the answers are limitless.

\section{Research Methodology}

Given the nature of the study it was decided to use Exploratory and Descriptive research design. Exploratory research provides acumens into a situation or an issue and is used where-

- There isn't a clear definition to the problem

- There is a genuine need to determine the best in class research design as well as best in class methods for data collection and subject selection

Descriptive research or Statistical research defines data and characteristics about the population or occurrence being studied. Descriptive research answers the questions who, what, where, when and how.

Thus, basis above, the two designs- Exploratory and Descriptive research designs were suitable for the present study as it was important to evaluate important parameters on the basis of which Agile projects could be judged.

The researcher also held focused group discussions to study Agile Software development within organizations. Participants in fo- cused group discussion were senior Delivery professionals (Delivery Head, Director, VP level) at three renowned organizations.

\section{Data Analysis}

There are certain projects where its difficult to apply agile development for instance-

- $\quad$ projects which are large in nature and those which involve agile development across multiple sites

- $\quad$ projects which have complex systems which are inter-dependent and

- $\quad$ projects with integrated hardware as well as software development.

These are the kind of issues which put agile implementations in doubt for large projects especially in industries like aerospace, banking etc. but at the same time making the implementation feasible for small projects [5].

A robust Systems Engineering Framework can help overcome such challenges. Another recipe for successful large scale agile development projects is a carefully and meticulously designed system architecture. It is also important at the same time to do the following-

- Perform incremental planning

- Run test cases using requirements which actually define the project especially within integration environments

- Create models which can help to facilitate Continuous Testing softwares which are related to each other and

- Organizational structure of the project to be in line with product architecture [5]. 


\section{Focused Group Discussions}

Three series of focused group discussions were held with 35 senior delivery professionals at three organizations- TCS, NIIT Technologies and Interglobe Technologies. The topics of discussion revolved around Limitations, Challenges, Situations/Scenarios where Agile would be a fit and Projects where Agile won't work Following are the key inferences-

\subsection{Difficulties/Challenges}

- Lack of interest from Leadership/Senior Management

- Communication related challenges

- $\quad$ Lack of training/Agile trained personnel [2]

\subsection{Limitations}

- Effective predictive control mechanisms can't be put to use [1]

- CMMi processes serve as anti-matter to Agile

- Steep learning curve

- Requirement of co-located teams

- Agile work on legacy systems

- Requirement of customer access

\subsection{Scenarios/Situations where Agile would be a good} fit

- Projects with ambitious deadlines

- Programs/Projects which have a very high element of complexity

- Unique projects/situations

- Projects having built-in uncertainty

\subsection{Projects where Agile might not work}

- Projects/Engagements of repetitive nature

- Projects in Infrastructure domain [4]

- Projects not having team members with prior exposure to Agile

- Projects not blessed by Senior Management

\section{Inference and Conclusion}

Agile software development has rightly received considerable attention lately. The agile approach can cut development lead times, reduce development costs, improve quality, and at the end of it all, increase customer satisfaction. Developers feel more in control of their work, get rapid feedback, are closer to the customer's needs and, as a result, avoid working on unnecessary activities. And with the right framework in place, agile development is not only possible for small, manageable projects but also for largescale projects.

However, success of many Agile projects does not necessarily guarantee Agile methodology to do wonders to all the projects irrespective of their nature. As came out during the Focused group discussions there are areas where Agile would certainly have an edge for example unique, uncertain projects with ambitious deadlines [3]. At the same time there are areas where Agile might not bring about any significant gains (given the time and effort that would be spent for its roll out) for example Infrastructure projects and projects of repetitive nature.

We should not get carried away by all the buzz created by Agile and must be prudent before arriving upon a decision. We should always reckon that software product development or application development is the destination and Agile as a tool could be a part of the journey that helps reach the destination depending on the internal as well as external factors.

\section{Conclusion}

We must perform a detailed analysis before selecting the project execution methodology and especially if we decide to choose Agile. We need to ask the right questions- Is the organization ready for Agile, does it have the culture and the mindset required which is so very necessary for Agile propagation and most important does it have the leadership buy-in and support.

\section{Acknowledgement}

Our sincere thanks to the leaders of organizations who patiently participated and gave honest views during the focused group discussion.

\section{References}

[1] A.T.Kearney (2013), Agile Software Hits the Major Leagues

[2] Dubakov Michael (2011), The future of Agile Software Development

[3] CA Technologies (2017), Indian Organizations are leading adoption of Agile in Asia Pacific

[4] Vijayasarathy, R (2008), Agile Software Development: a survey of early adopters

[5] Jalali S, Wohlin C (2010), Agile Practices in Global Software Engineering- A systematic Map 